\title{
Multimodality imaging of the left ventricle: Choosing soundly
}

\author{
Russell D. Rosenberg, MD, a and Pravin V. Patil, $M^{a}$ \\ a Section of Cardiology, Department of Medicine, Lewis Katz School of Medicine at Temple \\ University, Philadelphia, PA
}

Received Apr 23, 2018; accepted Apr 23, 2018

doi:10.1007/s12350-018-1294-8

\section{See related article, pp. 1857-1864}

Assessment of left ventricular systolic function by way of ejection function (LVEF) remains the foundation of cardiac imaging. Whether in the field of cardio-oncology, candidacy for device implantation, or left ventricular response to complex structural intervention, there is often no single piece of data more highly impactful on a patient's cardiovascular treatment and prognosis than LVEF. The expectation of the clinician, as well as the imaging tools locally available, will dictate what imaging modality may be ordered-often with multiple modalities selected, each informing the clinical scenario through their unique advantages and disadvantages (Figure 1).

In this issue, Pelletier-Galarneau et $\mathrm{al}^{1}$ describe the assessment of LVEF via IQ-SPECT GBPS and planar imaging in 60 patients. They compared the results to a small population of 11 patients assessed via cardiac MRI. Their data emphasize what we know as cardiovascular imagers: The accurate assessment of LV function is dependent on a range of variables including signal-to-noise ratio, temporal resolution, reproducibility of measurement, post-processing technique, and comparison to a gold-standard technique (Table 1).

Funding This research received no specific grant from any funding agency in the public, commercial, or not-for-profit sectors.

Reprint requests: Pravin V. Patil, MD, Section of Cardiology, Department of Medicine, Lewis Katz School of Medicine at Temple University, 9th Floor Parkinson Pavilion, 3401 N. Broad Street, Philadelphia, PA 19140; pravin.patil@tuhs.temple.edu

J Nucl Cardiol 2019;26:1865-8.

$1071-3581 / \$ 34.00$

Copyright (c) 2018 American Society of Nuclear Cardiology.

\section{ECHOCARDIOGRAPHY}

Echocardiography should be considered the mainstay of imaging for left ventricular function due to its ready availability, real-time imaging, excellent temporal resolution, and non-invasive nature. With minimal tissue effects, the negligible harm and lack of ionizing radiation exposure lends itself to serial evaluation of function. It is inherently tomographic from acquisition to interpretation with qualitative, semi-quantitative, and quantitative techniques. Semi-quantitative techniques are based on modeling of the LV with geometric assumptions that may be inappropriate. Quantitative techniques like real-time 3D echocardiography allow accurate assessment of LVEF compared to gold-standard cardiac MRI, contingent upon availability of acoustic windows. Acoustic window availability and susceptibility to artifacts remain its major limitation, though commercial contrast can often restore endocardial border definition and diagnostic accuracy.

\section{CARDIAC CT ANGIOGRAPHY}

Cardiac CT has not traditionally been used for assessment of LVEF. As it has become increasingly utilized by structural heart teams in addition to its role for coronary evaluation, the retrospectively gated data can be used to assess cardiac volumes accurately and determine LVEF. The cardiac volume is extracted from the dataset on the basis of attenuation and Hounsfield units (HUs). Though large multi-row and volumetric detectors allow whole heart single-beat scanning, the temporal resolution remains the key factor in data fidelity. The temporal resolution of cardiac CTA lies with the gantry rotation time, and recent iterations of equipment have seen the temporal resolution drop below $100 \mathrm{~ms}$. Arrhythmia can be significantly limiting in gated cardiac CTA, rendering studies non-diagnostic for $\mathrm{LV}$ function in some cases. 

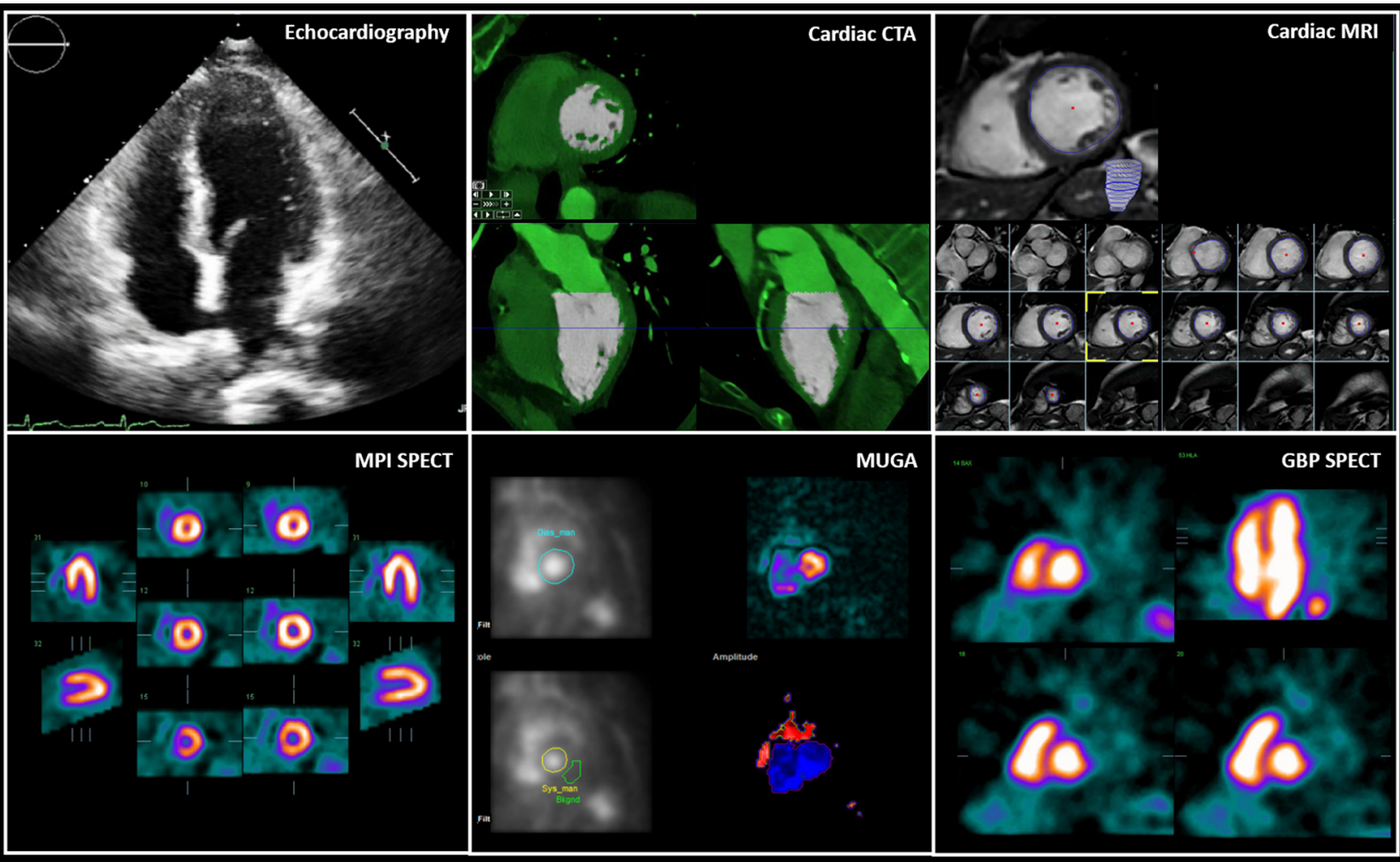

Figure 1. Functional assessment of the left ventricle.

\section{CARDIAC MAGNETIC RESONANCE}

Magnetic resonance imaging of the heart may not be readily available at all centers and can be a challenging tool to use in patients where serial followup is expected. However, its diagnostic accuracy has led it to become the gold-standard for volumetric assessment of ventricular systolic function. The most common technique is to use a method of disks summation through the short axis of the LV with steady-state free precession imaging. ${ }^{2}$ This allows for excellent endocardial definition to trace a cross-sectional area that can be multiplied by the slice thickness and added to determine cardiac volume and LVEF. This can overcome the geometric assumptions of semi-quantitative echocardiography. There are some anatomic considerations that come into play with cardiac MRI including definition of the final basal LV slice, whether to exclude papillary muscle anatomy, gating artifacts, and its need for consistent multiple breath-holds for diagnostic images. Cardiac MRI for LVEF does not necessitate contrast material use nor does it involve ionizing radiation, which leads to clinical appeal.

\section{SPECT MPI}

Myocardial perfusion imaging is often performed for a non-invasive risk stratification and ischemic evaluation. While not the primary focus of the study, LVEF can be assessed by first derivative analysis of count changes throughout the gating cycle. Without additional acquisition time or patient exposure, this datum coupled with perfusion imaging improves risk stratification. ${ }^{3}$ Gated MPI SPECT is susceptible to gating artifact, which can result in beat rejection if $\mathrm{R}$ $\mathrm{R}$ variability is excessive, predominately affecting enddiastolic imaging. MPI is also not focused on endocardial border imaging and errors in edge detection lead to errors in estimation of LVEF. Both the radiopharmaceutical used and the size and severity of an infarct can impact myocardial tracking and spatial resolution. ${ }^{4}$

\section{RADIONUCLIDE VENTRICULOGRAPHY}

\section{MUGA}

Multiple gated acquisition scanning (MUGA) has remained a traditional and common modality to assess LVEF over the past 40 years, often clinically implemented as the baseline and serial follow-up tool for chemotherapy-related cardiotoxicity. Utilizing a single 


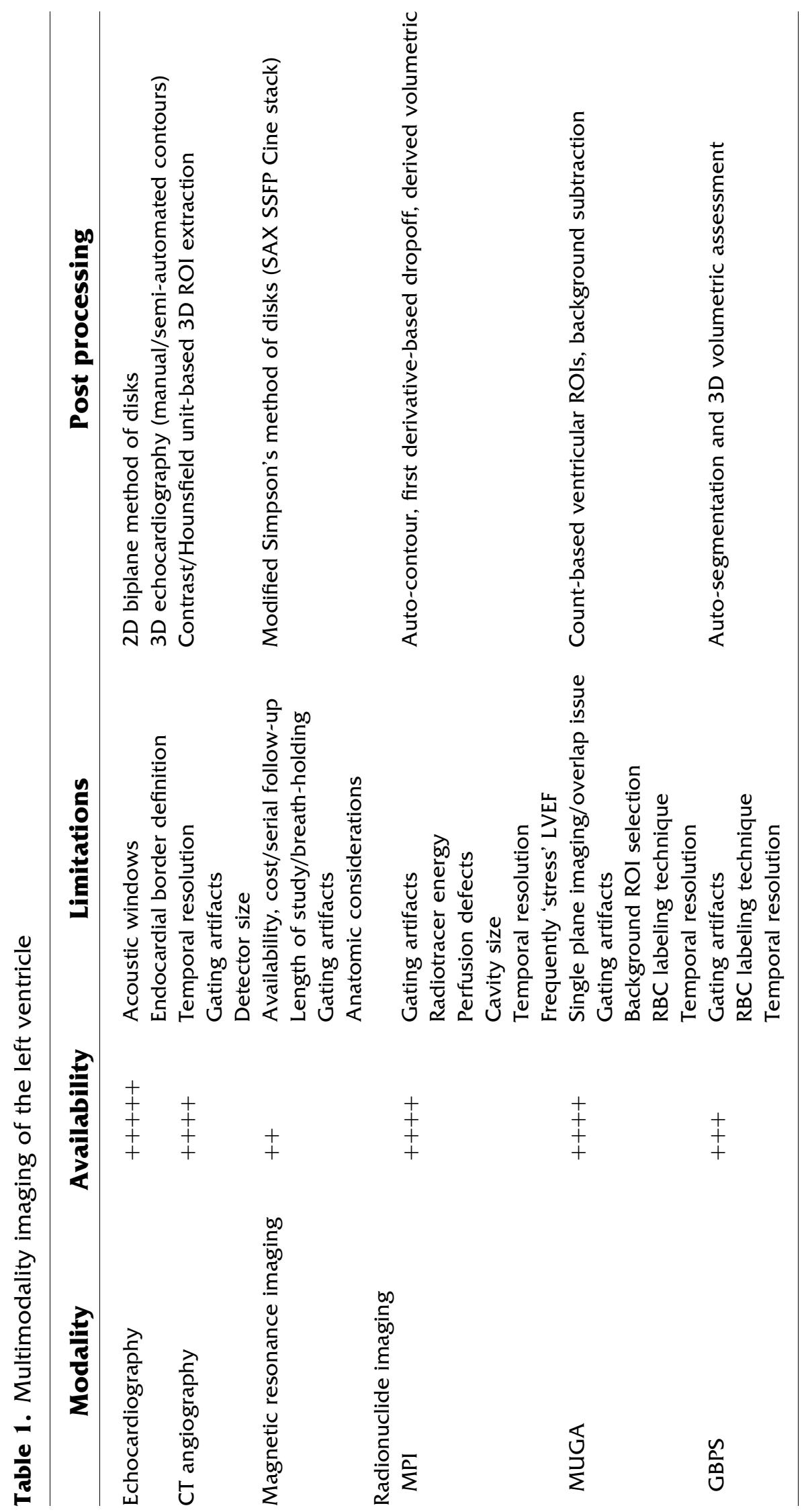


head gamma camera, there are inherent limitations including cardiac chamber overlap. This impacts accurate count assessment in differentiating the $\mathrm{LV}$ from adjacent chambers. ${ }^{5}$ MUGA involves identifying the LV region of interest (ROI) and background in a semiautomated background-subtracted fashion. Inappropriate selection of either of these ROIs can introduce measurement error in either direction. Count acquisition is summed over multiple cardiac cycles and remains susceptible to gating artifact. Erythrocyte labeling techniques will influence the count density of the blood pool.

\section{Gated Blood-Pool SPECT}

Advances in SPECT and acquisition technologies enhance diagnostic utility by employing gated bloodpool SPECT. Tomographic 3D acquisition of the blood pool (typical radiotracer, Tc-99m pertechnetate) allows the interpreting physician to distinguish both the RV and LV blood pool separately and analyze systolic function volumetrically. As highlighted by Pelletier-Galarneau et al, ${ }^{1}$ volume calculation, assessment of wall motion, and reduction in overlap limitations are clear advantages of GBPS compared to planar MUGA. Processing of GBPS data can be time consuming; while automated algorithms for LVEF assessment exist, manual reorientation of reconstructed data may still be required thus decreasing the repeatability of this technique. ${ }^{6,7}$ IQSPECT (Siemens Medical Solutions, Inc.) is a highsensitivity imaging solution employed for both GBPS and MPI SPECT study which offers advantages in shortening acquisition time and optimizing signal-tonoise ratio. This is largely achieved by using a multifocal collimator and cardiocentric orbit on a variable angle gamma camera. ${ }^{8}$ Imaging geometry creates unique attenuation artifact in IQ-SPECT, but this can be significantly reduced with $\mathrm{CT}$ attenuation correction. ${ }^{9,10}$

\section{SUMMARY}

Given the multimodality approach that clinicians can take to assess and validate LVEF, it is imperative that both referring and interpreting clinicians understand the advantages and disadvantages of each; specifically, how those align with an individual patient and clinical scenario. It is clear that the work by Pelletier-Galarneau et $\mathrm{al}^{1}$ further validates and enhances our understanding of the role IQ-SPECT and GBPS imaging play within the spectrum of assessment of left ventricular systolic function. Larger population studies of cardiac MRI and correlation with IQ-SPECT GBPS could help further validate this technique.

\section{Disclosure}

The authors reports no conflict of interest.

\section{References}

1. Pelletier-Galarneau M, Finnerty V, Tan S, Authier S, Gregoire J, Harel F. Assessment of left ventricular ejection fraction with cardiofocal collimators: Comparison between IQ-SPECT, planar equilibrium radionuclide angiography, and cardiac magnetic resonance. JNC 2018. https://doi.org/10.1007/s12350-018-1251-6.

2. Foley TA, Mankad SV, Anavekar NS, et al. Measuring left ventricular ejection fraction-Techniques and potential pitfalls. Eur Cardiol Rev 2012;8:108. https://doi.org/10.15420/ecr.2012.8.2. 108.

3. Hippeläinen E, Mäkelä $\mathrm{T}$, Kaasalainen $\mathrm{T}$, Kaleva E. Ejection fraction in myocardial perfusion imaging assessed with a dynamic phantom: Comparison between IQ-SPECT and LEHR. EJNMMI Phys 2017. https://doi.org/10.1186/s40658-017-0187-2.

4. Nichols K, DePuey EG, Krasnow N, Lefkowitz D, Rozanski A. Reliability of enhanced gated SPECT in assessing wall motion of severely hypoperfused myocardium: echocardiographic validation. J Nucl Cardiol 5:387-94. http://www.ncbi.nlm.nih.gov/pubmed/ 9715983.

5. Faber TL, Stokely EM, Templeton GH, Akers MS, Parkey RW, Corbett JR. Quantification of three-dimensional left ventricular segmental wall motion and volumes from gated tomographic radionuclide ventriculograms. J Nucl Med 1989;30:638-49. http:// www.ncbi.nlm.nih.gov/pubmed/2785584.

6. Van Kriekinge SD, Berman DS, Germano G. Automatic quantification of left ventricular ejection fraction from gated blood pool SPECT. J Nucl Cardiol 6:498-506. http://www.ncbi.nlm.nih.gov/ pubmed/10548145.

7. Wright GA, Thackray S, Howey S, Cleland JG. Left ventricular ejection fraction and volumes from gated blood-pool SPECT: Comparison with planar gated blood-pool imaging and assessment of repeatability in patients with heart failure. J Nucl Med 2003;44:494-8. http://www.ncbi.nlm.nih.gov/pubmed/12679390.

8. Nakajima K, Okuda K, Momose M, et al. IQ.SPECT technology and its clinical applications using multicenter normal databases. Ann Nucl Med 2017;31(9):649-59. https://doi.org/10.1007/ s12149-017-1210-3.

9. Caobelli F, Ren Kaiser S, Thackeray JT, et al. The importance of a correct positioning of the heart using IQ-SPECT system with multifocal collimators in myocardial perfusion imaging: A phantom study. J Nucl Cardiol 2015;22(1):57-65. https://doi.org/10. 1007/s12350-014-9994-1.

10. Gremillet E, Agostini D. How to use cardiac IQ•SPECT routinely? An overview of tips and tricks from practical experience to the literature. Eur J Nucl Med Mol Imaging 2016;43(4):707-10. https://doi.org/10.1007/s00259-015-3269-1. 